\title{
On the merger origin of nuclear star clusters
}

\section{Athanasia Tsatsi and Alessandra Mastrobuono-Battisti}

\author{
Max-Planck-Institut für Astronomie, Königstuhl 17, 69117 Heidelberg, Germany \\ email: tsatsi@mpia.de
}

\begin{abstract}
Nuclear Star Clusters (NSCs) are commonly observed in the centers of most galaxies, including our Milky Way (MW). While their study can reveal important information about the innermost regions of galaxies, the physical processes regulating their formation are still poorly understood. We explore a possible merger origin of NSCs by studying direct N-body simulations of globular clusters (GCs) that are initially randomly distributed in the outskirts and consecutively infall to the center of a MW-like nuclear bulge. We find that the NSC that forms through this process shows a significant amount of rotation, and both morphological and kinematic properties are comparable with observations of the MW NSC. We show that no finetuning of the orientation of the infalling GCs is necessary to result in a rotating NSC. This study shows the plausibility of the cluster infall scenario and can help towards setting better constraints to the formation history of NSCs.
\end{abstract}

Keywords. galaxies: nuclei, Galaxy: center, Galaxy: formation, globular clusters: general

\section{Introduction}

Over the last decade, a series of studies have shown that galaxies hosting Nuclear Star Clusters (NSCs) in their central regions are extremely common: including our own Milky Way, more than $77 \%$ of late type galaxies host NSCs (Böker et al. 2002), as well as at least $66 \%$ of early-type galaxies (Côté et al. 2006). With those fractions being only a lower limit to the true fraction of galaxies with NSCs, it has now been established that NSC formation must be a generic by-product of galaxy formation, making their study crucial in understanding the formation and secular evolution of their respective galaxy.

Two main scenarios have been proposed to explain the formation of NSCs; 1) the insitu formation model (e.g. Loose 1982), according to which the NSC forms as gas infalls into the center of the galaxy, where star formation takes place locally and most likely in an episodic manner (Schinnerer et al. 2008); and 2) the cluster infall scenario (e.g. Tremaine et al. 1975), where the NSC is formed by the accretion of globular clusters, that infall to the center due to dynamical friction. Both of these models are successful in explaining the mixture of stellar populations of different ages observed in NSCs (e.g. Rossa et al. 2006), and, until now, there has been no clear evidence of which model explains best the observations, or whether both of these processes could work in tandem towards the formation of NSCs.

Here, we explore how, and if, the cluster infall formation scenario can reproduce the observed morphological and kinematical properties of the Milky Way (MW) NSC, using N-body simulations of the consecutive infall of globular clusters (GCs) into a MW-like nucleus. We analyze the results in an observational-like manner, constructing mock photometric and kinematic maps that we use to assess the dynamical properties of the NSC. 


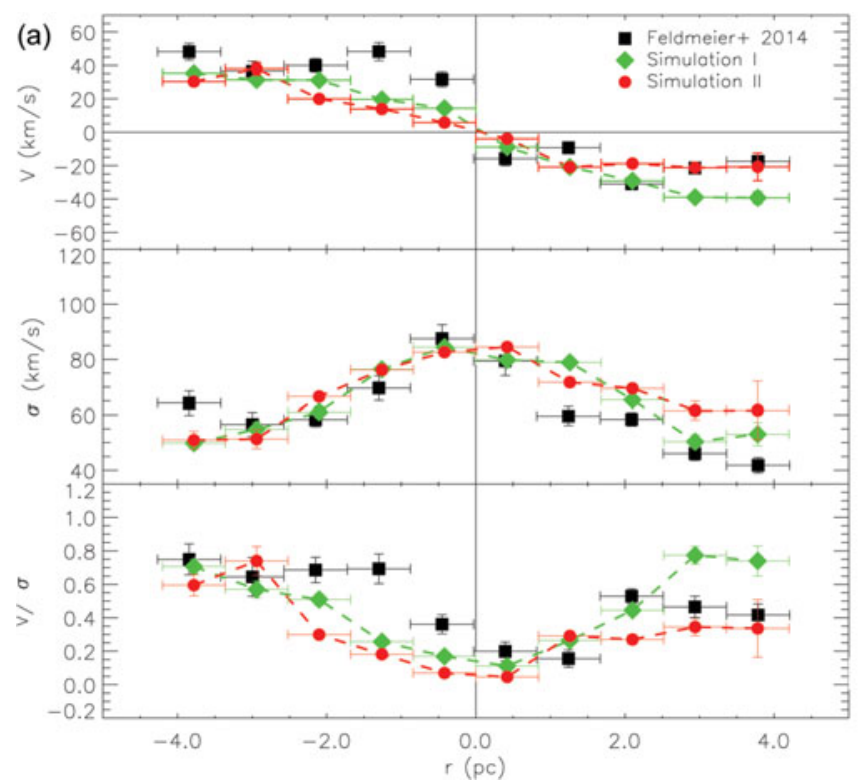

(b)
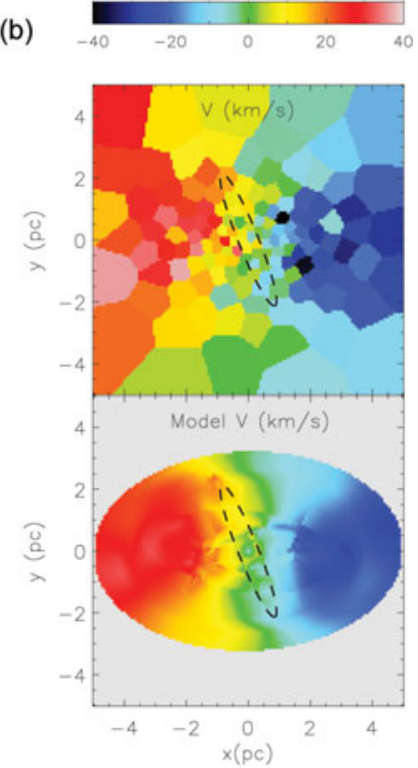

Figure 1. (a) Kinematic profiles of the Milky Way NSC (squares) as estimated by Feldmeier et al. (2014) and the corresponding profiles for our two simulations (dashed lines). (b) LOS velocity map of one of the simulations (top) and the corresponding kinemetric model (bottom). The overplotted ellipse shows the kinematic substructure.

\section{Simulations and mock kinematics}

The N-body simulations used in this work are described in detail in Antonini et al. (2012) and Perets et al. (2014). They simulate the formation of a NSC through the consecutive infall of $12 \mathrm{GCs}$, with random initial orbital parameters, into a nuclear bulge $\left(M_{n b}=10^{8} M_{\odot}\right)$, hosting a central massive black hole $\left(M_{\bullet}=4 \times 10^{6} M_{\odot}\right)$. The simulation lasts for $12.4 \mathrm{Gyr}$ and the total mass of the resulting NSC is approximately $1.5 \times 10^{7} M_{\odot}$, similar to the observed mass of the MW NSC (Genzel et al. 2010). In order to connect the orbital and mass distribution of the simulated NSC with observable properties, we create two-dimensional mock stellar mass and kinematic maps by projecting the stellar particles along a chosen viewing angle, spatially bin the maps using the 2D Voronoi binning method (Cappellari \& Copin 2003) and extract the mass-weighted Line-of-sight Velocity Distribution (LOSVD) of the cluster, which we fit with the Gauss-Hermite series.

\section{A direct comparison with observations}

The analysis of the extracted mock stellar mass and kinematic maps shows that the simulated NSC shows a significant amount of rotation and kinematic profiles (LOS velocity $\mathrm{V}$, velocity dispersion $\sigma$, and $V / \sigma$ ) that are comparable to the ones of the MW NSC (see Fig. 1(a) and Feldmeier et al. 2014). We show that two different simulations, that correspond to different (random) initial orbital parameters of the infalling clusters, result in NSCs with similar kinematic profiles. By analyzing the mock stellar maps, we find that the NSC also shows an amount of flattening (q 0.67) which is similar to the observed flattening of the MW NSC (Schödel et al. 2014). 


\section{Kinematic substructures as probes of the formation origin}

The detailed study of the internal kinematics can provide an important tool to dissentagle between possible formation mechanisms. Indeed, recent findings by Feldmeier et al. (2014) provide strong evidence for a kinematic substructure in the MW NSC, rotating perpendicularly to its main body, which can be interepreted as a fossil record of a past merger event. In order to study the role of mergers in creating such kinematic substructures, we use the Kinemetry method (Krajnović et al. 2006) in our simulated kinematic maps. We find a similar substructure (Fig. 1(b)), created by a past polar merger event of a globular cluster. This is a merger signature that can be observable and long-lasting (for $\sim 3$ Gyr) in the kinematics of the NSC.

\section{Conclusions}

We explore the possibility of a merger origin of NSCs, using N-body simulations of the consecutive infall of GCs in the center of a MW-like nucleus. We find that even if the GCs are initially randomly distributed around the center, the resulting NSC shows a significant amount of rotation, while both its morphological and kinematic properties are comparable to the MW NSC. Moreover, our adopted model can account for observable kinematic substructures in the final NSC, that can serve as long-lasting fossil records of past merger events. This is in line with recent observations of a similar substructure in the MW NSC (Feldmeier et al. 2014). We suggest that the cluster infall scenario is a viable hypothesis for NSC formation.

\section{Acknowledgements}

AT acknowledges financial support to the DAGAL network from the People Programme (Marie Curie Actions) of the European Union's Seventh Framework Programme FP7/2007- 2013/ under REA grant agreement number PITN-GA-2011-289313.

\section{References}

Antonini, F., Capuzzo-Dolcetta, R., Mastrobuono-Battisti, A., \& Merritt, D. 2012, ApJ, 750, 111

Böker, T., Laine, S., van der Marel, R. P., Sarzi, M., Rix, H.-W., Ho, L. C., \& Shields, J. C. 2002, AJ, 123, 1389

Cappellari, M. \& Copin, Y. 2003, MNRAS, 342, 345

Côté, P., Piatek, S., Ferrarese, L., Jordán, A., Merritt, D., Peng, E. W., Haşegan, M., Blakeslee, J. P., Mei, S., West, M. J., Milosavljević, M., \& Tonry, J. L. 2006, ApJS, 165, 57

Feldmeier, A., Neumayer, N., Seth, A., Schödel, R., Lützgendorf, N., de Zeeuw, P. T., KisslerPatig, M., Nishiyama, S., \& Walcher, C. J. 2014, A\&A, 570, A2

Genzel, R., Eisenhauer, F., \& Gillessen, S. 2010, Rev. of Modern Phys., 82, 3121

Krajnović, D., Cappellari, M., de Zeeuw, P. T., \& Copin, Y. 2006, MNRAS, 366, 787

Loose, H. H., Kruegel, E., \& Tutukov, A. 1982, A\&A, 105, 342

Perets, H. B. \& Mastrobuono-Battisti, A. 2014, ApJL, 784, L44

Rossa, J., van der Marel, R. P., Böker, T., Gerssen, J., Ho, L. C., Rix, H.-W., Shields, J. C., \& Walcher, C.-J. 2006, AJ, 132, 1074

Schinnerer, E., Böker, T., Meier, D. S., \& Calzetti, D. 2008, ApJL, 684, L21

Schödel, R., Feldmeier, A., Kunneriath, D., Stolovy, S., Neumayer, N., Amaro-Seoane, P., \& Nishiyama, S. 2014, AESA, 566, A47

Tremaine, S. D., Ostriker, J. P., \& Spitzer, Jr., L. 1975, ApJ, 196, 407

Tsatsi, A., \& Mastrobuono-Battisti, A., et al. 2016, in prep.

Walcher, C. J., van der Marel, R. P., McLaughlin, D., Rix, H.-W., Böker, T., Häring, N., Ho, L. C., Sarzi, M., \& Shields, J. C. 2005, ApJ, 618, 237 